\title{
A Survey on the Plant Leaf Disease Detection Techniques
}

\author{
Arpita Patel ${ }^{1}$, Mrs. Barkha Joshi ${ }^{2}$ \\ Student, Department of Computer Science, SVIT, Vasad, India ${ }^{1}$ \\ Assistant Professor, Department of Computer Science, SVIT, Vasad, India ${ }^{2}$
}

\begin{abstract}
Plant disease detection is an interesting field in India a. Plants are the way to live. We are completely dependent on plants from our daily life factors to breathing. So, there should be proper care of plants. Many studies show that quality of agricultural products may be reduced due to various factors. The plant diseases are such as fungi, bacteria, and viruses. The leaf diseases not only restrict the growth of the plant but also destroy its crop. There is the need of some expert to identify plant diseases but manual identification is time consuming. So, some automatic methods required. In this paper, we have presented a survey on the existing methods of plant leaf disease detection.
\end{abstract}

Keywords: Image Processing, Leaf Disease, Feature Extraction, Disease Identification.

\section{INTRODUCTION}

Plant disease diagnosis is an art as well as science. The diagnostic process (i.e. recognition of symptoms and $\mathrm{A}$ signs), is inherently visual and requires intuitive judgement as well as the use of scientific methods. India is a cultivated country and about $70 \%$ of the population depends on agriculture. Disease on plant leads to the significant reduction in both the quality and quantity of agricultural products. The studies of plant disease refer to the studies of visually observable patterns on the plants. Monitoring of disease on plant plays an important role in successful cultivation of crops in the farm. In early days, the monitoring and analysis of plant diseases were done manually by the expertise person in that field. This requires large amount of work and also requires excessive processing time. The image processing techniques can be used in the plant disease detection [1].

The detection and classification of leaf diseases accurately is the key to prevent the agriculture loss. Different plant leaf bears different diseases. There are a list of methods and classifiers to detect plant leaf diseases. The considered methods for plant leaf diseases are explained as existing work in section IV.

Section II describes the basic concept of leaf diseases symptoms. Section III explains the literature reviews. Section V concludes the paper.

\section{LEAF DISEASES \& SYMPTOMS}

Leaves are mainly affected by bacteria, fungal and viral. A symptom of plant disease is a visible e effect of disease on the plant. Symptoms may include a detectable change in color, shape or function of the plant as it responds to the pathogen. Here, we are discussing these diseases symptoms that should be keep in mind if plant growth seems low.
A. Viral Disease Symptoms

Among all plant leaf diseases, those caused by viruses are the most difficult to diagnosis. Viruses produce no telltale signs that can be readily observed and often confused with nutrient deficiencies and herbicide injury. Aphids, leafhoppers, whiteflies and cucumber beetles insects are common carriers of this disease, e.g. Mosaic virus, and look for yellow or green stripes or spots on foliage, as shown in Fig. 1. Leaves might be wrinkled, curled and growth may be stunted [7].

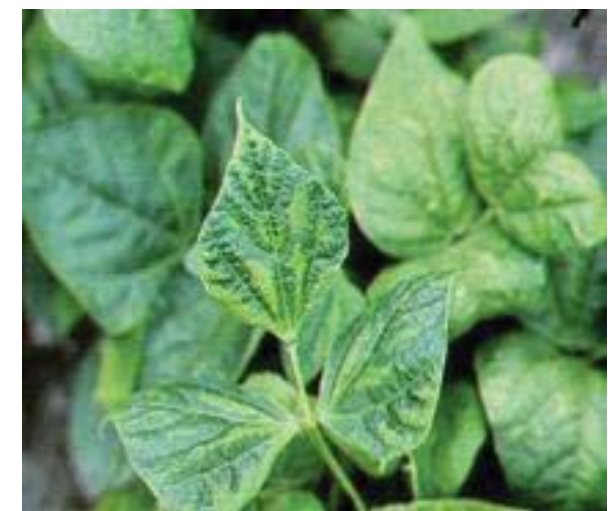

Figure 1: Viral Disease Symptoms

\section{B. Bacterial Disease Symptoms}

Pathogenic bacteria cause many serious diseases of vegetables. They do not penetrate directly into plant tissue but need to enter through wounds or natural plant openings. Wounds can result from damage by insects, other pathogens, and tools during operations such as pruning and picking

The disease is characterized by tiny pale green spots which soon come into view as water- soaked. The lesions enlarge and then appear as dry dead spot as shown Fig. 2. 
Vol. 6, Issue 1, January 2017

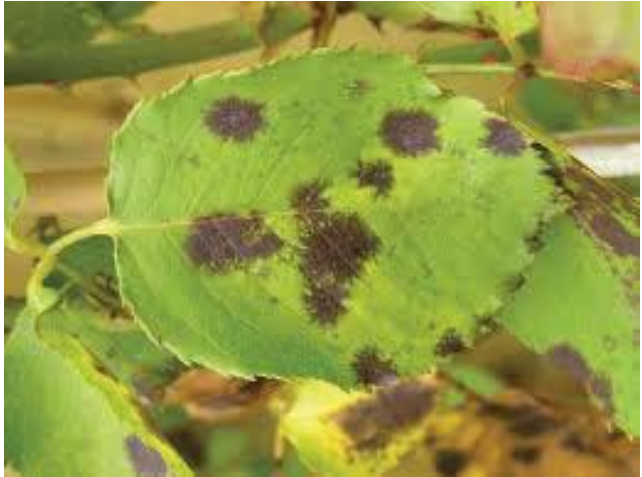

Figure 2: Bacterial Disease Symptoms

\section{Fungal Disease Symptoms}

Plant leaf diseases, those caused by fungus are discussed below and shown in Fig. 3. E.g. Late blight caused by fungus. It first appears on lower, older leaves like graygreen spots, water- soaked. When fungal disease matures, these spots darken and then white fungal growth forms on the undersides.

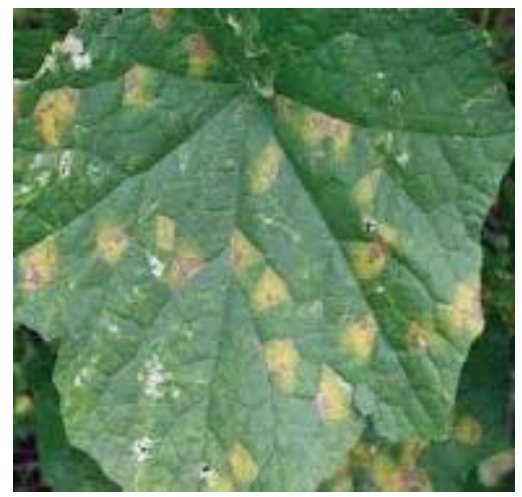

Figure 3: Fungal Disease Symptoms

\section{III.LITERATURE SURVEY}

Khirade et al. [3] has discussed some segmentation and feature extraction algorithm that can be used for the detection of plant diseases by using the image of their leaves. It is very difficult to detect the plant diseases manually due to requirement of excessive time, knowledge of plant diseases and much amount of work. The author has divided the entire process of plant leaf diseases detection into five steps: Image acquisition, Preprocessing, Segmentation, Feature extraction and Final classification of diseases. Image acquisition used the transformation structure for RGB leaf image. Then image is pre-processed to remove the noise and enhance the image contrast. Segmentation is done for the partitioning of image into various feature parts using k-means clustering, otsu filters etc. This segmented image is further used for feature extraction and then final classification is performed using various classification techniques. In this way, plant diseases can be efficiently identified.

Sannakki et al. [4] has used feed forward back propagation Neural Network based technique for the diagnosis and classification of diseases in grape leaf. Author has used the image s of grape leaf with complex background for the diagnosis as input. Further anisotropic diffusion is used to remove the noise of the image which is further segmented using k-means clustering. Finally results are observed using neural network. Results are experimented on downy mildew and powdery mildew images with simulation in MATLAB. Confusion matrix is considered with the true positive and false positive parameters for the validation of results. The author claimed to have the training accuracy of $100 \%$ if used hue feature alone.

Kutty et al. [5] has used the neural network based system to classify the watermelon leaf diseases of Downey Mildew and Anthracnose. Author has calculated the true positive rate, true negative rate and overall accuracy for the efficiency of the proposed concept This classification is based on the color feature extraction from RGB color model which is obtained from the identified pixels in the region of interest.. The overall performance is depicted with ROC curve having AUC value of 0.5 . The true classification result also depicts the value of $75.9 \%$.

Rothe et al. [6] has proposed pattern recognition techniques for the detection and classification of cotton leaf diseases of Alternarnia, Myrothecium and Bacterial Blight. The dataset images are taken from the field of Central Institute of Cotton Research Nagpur. Active contour based segmentation algorithm is used for the iolation of diseased spots. Author has also suggested some feature directions to the similar concept for the crops of wheat, orange, citrus and maize etc.

\section{TABLE 1: COMPARISON OF DIFFERENT TECHNIQUES}

\begin{tabular}{|c|c|c|}
\hline Author Name & Techniques & Description \\
\hline $\begin{array}{ll}\text { Sachin } & \text { D. } \\
\text { Khirade, } & \\
\text { A.B. Patil } & \end{array}$ & $\begin{array}{l}\text { K-means } \\
\text { clustering }\end{array}$ & $\begin{array}{l}\text { Discussed various } \\
\text { plant leaf diseases } \\
\& \text { classification } \\
\text { method }\end{array}$ \\
\hline Sannakki et al. & $\begin{array}{l}\text { Feed } \\
\text { Forward } \\
\text { Back } \\
\text { Propagation } \\
\text { Neural } \\
\text { network }\end{array}$ & $\begin{array}{l}\text { Neural network } \\
\text { based classification } \\
\text { is performed for } \\
\text { the grape leaf } \\
\text { diseases detection. }\end{array}$ \\
\hline Kutty et al. & $\begin{array}{l}\text { Neural } \\
\text { Network }\end{array}$ & $\begin{array}{l}\text { Neural network is } \\
\text { used to classify } \\
\text { watermelon leaf } \\
\text { disease } \\
\text { accuracy } 75.9 \%\end{array}$ \\
\hline $\begin{array}{l}\text { P.R. Rothe, } \\
\text { R.V. } \\
\text { Kshiragar }\end{array}$ & $\begin{array}{l}\text { Neuro- } \\
\text { Fuzzy } \\
\text { Inference } \\
\text { System }\end{array}$ & $\begin{array}{lr}\text { Cotton } & \text { leaf } \\
\text { diseases } & \text { of } \\
\text { Alternarnia, } & \\
\text { Myrothecium } & \text { and } \\
\text { Bacterial } & \text { are } \\
\text { detected. } & \\
\end{array}$ \\
\hline
\end{tabular}


Vol. 6, Issue 1, January 2017

\section{IV.BASIC CONCEPT OF LEAF DISEASE DETECTION}

Plant leaf disease detection includes some basic step of image processing to detect \& classify plant leaf disease. These steps are image acquisition, image pre-processing, image segmentation, feature extraction, classification and leaf disease detection. These steps are described as below in figure 1.

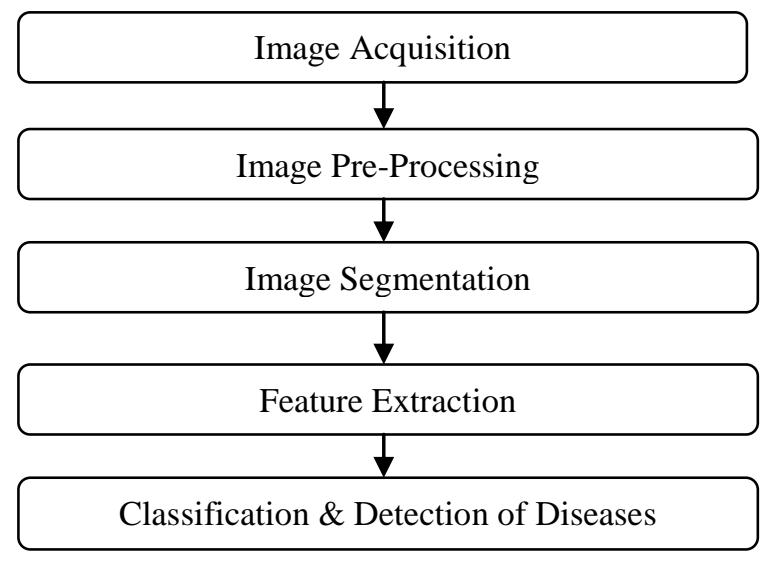

Figure 1: Basic concept of leaf disease detection

\section{A. Image Acquisition}

The first stage of any vision system is the image acquisition. Image acquisition involves the steps to obtain the plant leaf and captured the high quality images through the camera. Images are acquired from the internet or agriculture field. The efficiency of the concept depends upon the quality of database images. This image is in RGB (Red, Green, and Blue) form.

\section{B. Image Pre-Processing}

Image pre-processing involves the steps of image enhancement, RGB to Lab conversion, filtering etc. Here, image enhancement is carried out for increasing the contrast. Image smoothing is done using the filtering techniques. There are different types of filtering techniques available in image processing like median filter, average filter, Gaussian filter etc.

\section{Image Segmentation}

Image segmentation means partitioning of image into various parts of same features or having some similarity. The segmentation can be done using various methods like otsu' method, k-means clustering, converting RGB image into HIS model etc. The K-means clustering is used for classification of object based on a set of features into $\mathrm{K}$ number of classes. The classification of object is done by minimizing the sum of the squares of the distance between the object and the corresponding cluster.

\section{Feature Extraction}

Feature extraction plays an important role for identification of an object. After performing the image segmentation the disease portion from the image is extracted. In many application of image processing feature extraction is used. Color, texture, shape, edges, morphology are the features which can be used in plant disease detection. Color features are extracted by various methods, such as Color histogram, Color moments and Color structure descriptor. Grey Level Co-occurrence Matrix (GLCM) method is used for extraction of texture features [8].

\section{E. Classification \& Detection of Diseases}

Finally, classifiers are used for the training and testing of the datasets. These classifiers may be support vector machine (SVM), k-nearest neighbour, neural network, fuzzy logic based etc. These methods are used to classify and detect the leaf diseases.

\section{CONCLUSION}

In this paper, we have discussed various methods for the identification and classification of plant leaf diseases like pattern recognition method, back propagation, neural network, support vector machine etc. We have also discussed the basic concept of plant leaf disease detection and various leaf diseases symptoms.

\section{REFERENCES}

[1] Arivazhagan, S., R. Newlin Shebiah, S. Ananthi, and S. Vishnu Varthin. "Detection of unhealthy region of plant leaves and classification of plant leaf diseases using texture features." Agriculture Engineering International: CIGR Journal 15, No.1 (2013): 211-217.

[2] Kiran R. Gavhale and Ujwalla Gawande. "An Overview of the Research on Plant Leaves Disease detection using Image Processing Techniques." IOSR Journal of Computer Engineering, January 2014.

[3] Sachin D. Khirade and A. B. Patil. "Plant Disease Detection Using Image Processing." International Conference on Computing Communication Control and Automation (ICCUBEA), 2015 International Conference on,pp. 768-771. IEEE, 2015.

[4] Sannakki, Sanjeev S., Vijay S. Rajpurohit, V. B. Nargund, and Parag Kulkarni. "Diagnosis and classification of grape leaf diseases using neural networks." In computing, communications and Networking Technologies (ICCCNT), 2013 Fourth International conference on, pp. 1-5 IEEE, 2013.

[5] Kutty, Suhaili Beeran, Noor Ezan Abdullah, Habibah Hashim, and Aida Sulinda. "Classification of Watermelon Leaf Diseases Using Neural Network Analysis.” In Business Engineering and Industrial Applications Colloquium (BELAC), 2013 IEEE, pp. 459-464. IEEE, 2013.

[6] Rothe, P. R., and R. V. Kshirsagar. "Cotton Leaf Disease Identification using Pattern Recognition Techniques.” In Pervasive Computing (ICPC), 2015 International Conference on, pp. 1-6. IEEE, 2015.

[7] Pearson, Roger C., and Austin C. Goheen. Compendium of leaf diseases, American Phytopathological Society, 1988.

[8] I. EI Massi, Y. Es-saady, M. EI Yassa, D. Mammass and A. Benazoun. "Automatic recognition of plant leaves diseases based on serial combination of two SVM classifiers." 2nd International Conference on Electrical and Information Technologies (ICEIT), IEEE, 2016.

[9] Aakanksha Rastogi, Ritika Arora and Shanu Sharma. "Leaf Disease Detection and Grading using Computer Vision Technology \&Fuzzy Logic." 2nd International Conference on signal processing \& Integrated Network (SPIN), IEEE, 2015.

[10] Garima Tripathi. "Review on color and texture feature extraction techniques." International Journal of Enhanced Research in Management \& Computer Applications, Vol. 3 Issue 5, pp. 77-81, May-2014. 